\title{
Meridian Observations and Elements of Comet Wells.
}

The place of the comet at lower culmination was compared, by means of the Pistor and Martins circle, with stars preceding and following on nearly the same parallel of declination. When the stars selected from $\$ 539$ Sterne* proved insufficient, special star positions were computed from a combination of good modern authorities. The ob- servations were reduced by assistant $R$. H. Tucker jr. and compared with places specially computed by him from elements by C. S. Wells, given below. The Albany mean times are corrected for aberration. The declinations have also been corrected for parallax, and the quantity used is given in the column following:

\begin{tabular}{|c|c|c|c|c|c|c|c|}
\hline 1882 & Albany $\mathrm{m} . \mathrm{t}$. & $\alpha$ app. & $\delta$ app. & Parallax & $\begin{array}{l}\text { Corr. } \\
\Delta \alpha\end{array}$ & $\begin{array}{r}\text { o eph. } \\
\Delta \delta\end{array}$ & Observer \\
\hline May 15 & $9^{h} 4^{8^{m}} 24^{s} \cdot 0$ & $I^{h} 30^{m} 44^{s} \cdot 62$ & $+72^{\circ} 41^{\prime} 54^{\prime \prime} 3$ & $+8: 9$ & +.54 & -0.7 & Boss. \\
\hline 16 & $10 \quad 4 \quad 5^{8.4}$ & I $5 \mathrm{I} \quad \mathrm{I} 6.84$ & +714825.0 & +9.0 & +.50 & -5.8 & Tucker. \\
\hline 23 & $\begin{array}{lll}\text { I } & 14 & 19.7\end{array}$ & $\begin{array}{lll}3 & 28 & 23 \\
3\end{array}$ & +623441.7 & +9.6 & +.32 & -1.8 & Boss. \\
\hline 25 & I I 2337.1 & $\begin{array}{lll}3 & 45 & 7.42\end{array}$ & $\begin{array}{r}+59184.8 \\
\end{array}$ & +9.7 & +.33 & -0.7 & 'Tucker. \\
\hline 26 & II $26 \quad 18.9$ & 35217.99 & +573512.0 & +9.7 & +30 & -2.9 & Tucker. \\
\hline
\end{tabular}

The interval from May 16 to May 23 was almost continuously cloudy. The elements used in the comparisons were computed by Assistant C.S. Wells and appear to be accurate. The Albany filar-micrometer observations used by Mr. Wells are as follow:

\begin{tabular}{|c|c|c|c|c|c|c|c|c|c|c|}
\hline 1882 & Albany $m . t$ & $\Delta \alpha$ & $\Delta d^{\prime}$ & Comp. & $\alpha$ app. & $\log p .4$ & $\delta$ app. & $\log p . \Delta$ & Obs. & * \\
\hline $\operatorname{arch} \times 9$ & $\mathrm{I} 3^{\mathrm{h}} 27^{\mathrm{m}} 18^{\mathrm{s}}$ & $+1^{m} 3^{85} \cdot 76$ & $+2{ }^{\prime} z^{\prime \prime} \cdot 5$ & 27.9 & $17^{\mathrm{h}} 54^{\mathrm{m}} 45^{\mathrm{s}} 53$ & $9.686_{n}$ & $+33^{\circ} 27^{\circ} 23^{\prime} 2$ & 0.566 & $\left.B^{*}\right)$ & \\
\hline 19 & I5 I 8 II & + I 46.55 & +58.1 & $9 \cdot 3$ & I 75453 & & +332949.0 & 0.354 & B & \\
\hline April & I0 4045 & $+\circ 44.54$ & $+\circ 39.5$ & I 2.6 & $18 \quad 52 \quad 18$. & & +514452.0 & 0.6 & $\mathbf{E}$ & \\
\hline 16 & $\begin{array}{lll}9 & 46 & 23\end{array}$ & +10.10 & 一 00.7 & 20.10 & $\begin{array}{lll}18 & 59 & 12.58\end{array}$ & $9.82 \mathrm{O}_{\mathrm{n}}$ & +533447.0 & 0.764 & $\mathrm{E}$ & \\
\hline 16 & I0 3327 & - I 10.01 & +010.7 & I 8.9 & $\begin{array}{lll}18 & 59 & 19.63\end{array}$ & $9.852 \mathrm{n}$ & $+533^{6} 3^{6.9}$ & 0.680 & E & \\
\hline 17 & 101050 & -015.90 & 一 I 35.5 & I 2.6 & $\begin{array}{lll}19 & 3 & 3.40\end{array}$ & $9.842_{n}$ & $+543^{2} 54.6$ & 0.735 & $\mathbf{E}$ & \\
\hline May & $10 \begin{array}{lll}0 & 3 & 3\end{array}$ & -221.06 & -433.3 & 30.10 & I $30 \quad 50.96$ & - & +724132.6 & 0.997 & $\mathrm{E}$ & 6 \\
\hline
\end{tabular}

*) $\mathrm{B}=$ Boss; $\mathrm{E}=$ Egbart.

Comparison Stars.

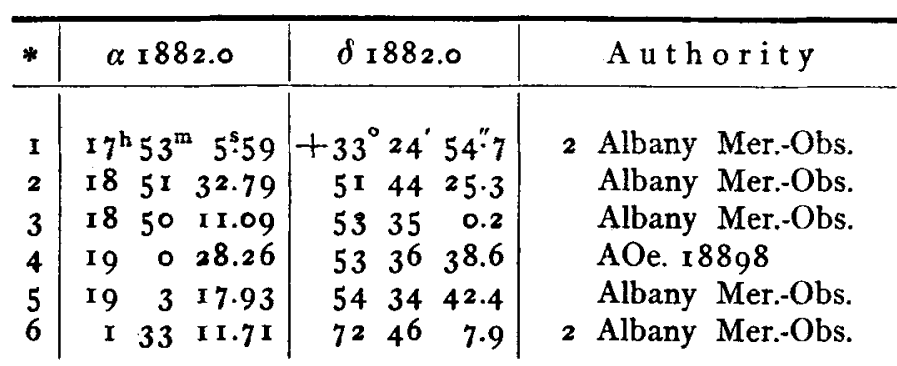

To these were added the Albany meridian observation of May 15; Kiel April 14; and Paris April 14, 16. The resulting elements are:

$$
\begin{aligned}
& T=\mathrm{r} 882 \text { Juni } 10.530055 \text { Greenwich } \mathrm{m}, \mathrm{t} \text {. } \\
& \pi-\Omega=208^{\circ} 59^{\prime} 29^{\prime \prime} 6 \\
& \delta=204 \quad 56 \quad 16.8 \text { Ecliptic and Equin. I } 882.0 \\
& i=\begin{array}{lll}
73 & 48 & 32.3
\end{array} \\
& \log q=8.7837 \times 99 \text {. }
\end{aligned}
$$

$$
\begin{aligned}
& \text { Middle place }(\mathrm{C}-\mathrm{O}) \\
& \Delta \lambda \cos \beta=-2.6 \\
& \Delta \beta=+1.0
\end{aligned}
$$

Equations of coordinates.

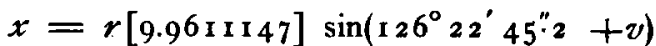

$$
\begin{aligned}
& y=r[9.8608394] \sin (6 \text { I I I } 35.7+v) \\
& z=r[9.9021089] \sin (1965116.3+v)
\end{aligned}
$$

Dudley Observatory 1882 June $\mathbf{r}$.

Lewis Boss.

\section{Ueber die Periode des veränderlichen Sterns DM. $+1^{\circ} 3408$.}

Im Dun Echt Circular No. 53 findet sich folgende Nachricht über obigen Stern, dessen Veränderlichkeit von Herrn Edw. F. Sawyer in A. N. Bd. ror pag. 187 angezeigt ist. Die dort angegebene Periode beträgt $5^{1 / 4}$ Tage.

-A Science Observer telegram announces that Mr. S. C. Chandler, Jr. finds the period of the variable star DM. $+1^{\circ} 3408$ to be only 0.83 days. A minimum occurs on July $i, 12^{\mathrm{h}} 57^{\mathrm{m}} \cdot \mathrm{G}$. M. T. 